\title{
ISOPERIMETRIC INEQUALITIES FOR PARAMETRIC VARIATIONAL PROBLEMS
}

\section{INÉGALITÉS ISOPÉRIMÉTRIQUES POUR LES PROBLÈMES VARIATIONNELS PARAMÉTRIQUES}

\author{
UIrich CLARENZ ${ }^{\text {a }}$, Heiko VON DER MOSEL ${ }^{\text {b }}$ \\ ${ }^{a}$ Fachbereich Mathematik, Gerhard-Mercator-Universität Duisburg, Lotharstraße 63/65, \\ 47048 Duisburg, Germany \\ $\mathrm{b}^{\mathrm{b}}$ Mathematisches Institut, Universität Bonn, Beringstraße 1, 53115 Bonn, Germany
}

Received 26 February 2001

\begin{abstract}
We prove isoperimetric inequalities for general parametric variational double integrals $\mathcal{F}$, whose Lagrangians $F$ depend on the position vector $X$ and on the surface normal $N$. As an essential tool we introduce Sauvigny's $F$-conformal parameters adapted to the parametric integrand and use the notion of generalized mean and Gaussian curvature. The special cases of minimal surfaces, surfaces of bounded mean curvature and $\mathcal{F}$-minimizing surfaces are also discussed.

(C) 2002 L'Association Publications de l'Institut Henri Poincaré. Published by Elsevier B.V. All rights reserved

RÉSUMÉ. - Nous démontrerons des inégalités isopérimétriques pour les intégrales doubles variationnelles paramétriques générales $\mathcal{F}$ dont la fonction de Lagrange $F$ dépend du vecteur de position $X$ et de la surface normal $N$. Comme outil essentiel, nous introduiserons des paramètres $F$-conformes de Sauvigny adaptés l'intégrand paramétrique et nous employerons la notion de la courbure moyenne généralisée et gaussienne généralisée. Nous discuterons également les cas particuliers des surfaces minimales, des surfaces de courbure moyenne limitée et des surfaces minimalisant $\mathcal{F}$.

(C) 2002 L'Association Publications de l'Institut Henri Poincaré. Published by Elsevier B.V. All rights reserved
\end{abstract}

\section{Introduction}

We consider general parametric functionals of the form

$$
\mathcal{F}(X):=\int_{B} F\left(X, X_{u} \wedge X_{v}\right) d u d v
$$

E-mail addresses: clarenz@math.uni-duisburg.de (U. Clarenz), heiko@math.uni-bonn.de (H. von der Mosel). 
where $B \subset \mathbb{R}^{2}$ is the open unit disk in the plane. The Lagrangian $F$ is of class $C^{0}\left(\mathbb{R}^{3} \times \mathbb{R}^{3}\right) \cap C^{3, \alpha}\left(\mathbb{R}^{3} \times\left(\mathbb{R}^{3}-\{0\}\right)\right.$ for some $\alpha \in(0,1)$, and satisfies the homogeneity condition

$$
F(y, t z)=t F(y, z) \quad \text { for all } t>0 \text { and }(y, z) \in \mathbb{R}^{3} \times \mathbb{R}^{3} .
$$

Moreover, we assume that $F$ is elliptic, i.e., that the mapping $F_{z z}(y, z): z^{\perp} \rightarrow z^{\perp}$ is positive definite for all $(y, z) \in \mathbb{R}^{3} \times\left(\mathbb{R}^{3}-\{0\}\right)$, where ${ }^{1}$

$$
z^{\perp}=\left\{\zeta \in \mathbb{R}^{3} \mid\langle\zeta, z\rangle=0\right\}
$$

Since $F_{z z}$ is homogeneous of degree -1 in its second argument by $(\mathrm{H})$, this implies that there exist constants $0<M_{1} \leqslant M_{2}<\infty$ such that

$$
M_{1}\left|P_{z^{\perp}} \xi\right|^{2} \leqslant|z|\left\langle\xi, F_{z z}(y, z) \xi\right\rangle \leqslant M_{2}\left|P_{z^{\perp}} \xi\right|^{2}
$$

for all $(y, z) \in \overline{B_{1}(0)} \times\left(\mathbb{R}^{3}-\{0\}\right), \xi \in \mathbb{R}^{3}$, where $P_{z \perp} \xi=\xi-\langle\xi, z\rangle z /|z|^{2}$ is the projection onto $z^{\perp}$ and where $B_{1}(0)$ denotes the open unit ball in $\mathbb{R}^{3}$.

In addition, we suppose that $X$ is an immersed surface of class $C^{2, \alpha}\left(\bar{B}, \mathbb{R}^{3}\right)$ that maps the boundary $\partial B$ topologically onto a given closed Jordan curve $\Gamma \subset \mathbb{R}^{3}$ with length $L(\Gamma)$. The aim of the present paper is to prove an isoperimetric inequality for immersions $X$ that are $\mathcal{F}$-critical, i.e., stationary for the parametric functional (1). To be more precise we are going to estimate the area

$$
\mathcal{A}(X):=\int_{X} d A=\int_{B}\left|X_{u} \wedge X_{v}\right| d u d v
$$

in terms of quantities depending only on $\Gamma$ and $F$. The area functional $\mathcal{A}$ itself is a special parametric functional of the form (1) with the integrand $F(y, z)=A(z):=|z|$. It is a well-known fact that $\mathcal{A}$-critical surfaces, i.e., minimal surfaces of the type of the disk satisfy the classical isoperimetric inequality

$$
\mathcal{A}(X) \leqslant \frac{1}{4 \pi} L^{2}(\Gamma)
$$

see e.g. [6, Ch. 6.3]. Note that this is true for all surfaces with nonpositive Gauß curvature $K$ (cf. $[2,1]$ ). More generally, disk-type surfaces of prescribed bounded mean curvature $H$, whose Gauß curvature may have varying sign, can be obtained as critical points of the parametric functional with the integrand

$$
F(y, z)=E(y, z):=|z|+\langle Q(y), z\rangle,
$$

where $Q$ is a weakly differentiable vector field on $\mathbb{R}^{3}$ with $\operatorname{div} Q=H$ on $\mathbb{R}^{3}$, compare with Hildebrandt [8]. Under the assumption that

$$
\|X(.)\|_{\infty, \bar{B}}:=\sup _{w \in \bar{B}}|X(w)| \leqslant 1 \quad \text { and } \quad h:=\|H(.)\|_{\infty, \mathbb{R}^{3}}<2,
$$

${ }^{1}$ Note that $(\mathrm{H})$ implies $F_{z z}(y, z) z=0$ for all $(y, z) \in \mathbb{R}^{3} \times\left(\mathbb{R}^{3}-\{0\}\right)$. 
one can show that

$$
\mathcal{A}(X) \leqslant \frac{1}{8(1-(h / 2))} L^{2}(\Gamma)
$$

for such surfaces, which is a result of Heinz and Hildebrandt [7]. ${ }^{2}$ In [4] we generalized (3) to critical immersions of parametric functionals with an integrand $F(y, z)=F(z)$ depending only on $z$, which does not cover the case of surfaces of prescribed bounded mean curvature, cf. (4). In the present work we are going to prove the following isoperimetric inequality for critical immersions of general parametric double integrals (1), which contains all the previous cases.

THEOREM 1.- Let $X \in C^{2, \alpha}\left(\bar{B}, \mathbb{R}^{3}\right), \alpha \in(0,1)$, be an $\mathcal{F}$-critical immersion with surface area $\mathcal{A}(X)$, which maps $\partial B$ topologically onto a closed Jordan curve $\Gamma$. Assume that the parametric elliptic integrand $F=F(y, z)$ is of class $C^{0}\left(\mathbb{R}^{3} \times \mathbb{R}^{3}\right) \cap C^{3, \alpha}\left(\mathbb{R}^{3} \times\right.$ $\left.\left(\mathbb{R}^{3}-\{0\}\right)\right)$, and set $\left\|F_{y z}\right\|:=\left\|F_{y^{i} z^{i}}(., .)\right\|_{\infty, \overline{B_{1}(0)} \times S^{2}}$. Then there is a constant $C=C(F)$ depending solely on $F$, such that if

$$
\begin{gathered}
\|X(.)\|_{\infty, B} \leqslant 1, \\
h_{F}:=\left[C(F)\left(1+\left\|F_{y z}\right\|^{2}\right)+\left(\left\|F_{y z}\right\| / M_{1}\right)\right]<2,
\end{gathered}
$$

then

$$
\mathcal{A}(X) \leqslant \frac{2 C(F) \frac{M_{2}}{M_{1}}\left[\int_{\Gamma} k d s-2 \pi\right] L(\Gamma)+\sqrt{\frac{M_{2}}{M_{1}}} L^{2}(\Gamma)}{8\left(1-\left(h_{F} / 2\right)\right)} .
$$

If $F(y, z)=A(z)=|z|$, or if $F(y, z)=E(y, z)=|z|+\langle Q(y), z\rangle$ for some $Q \in$ $W_{\text {loc }}^{1, \infty}\left(\mathbb{R}^{3}, \mathbb{R}^{3}\right)$, then $C(F)=0$ and $M_{1}=M_{2}=1$.

Remarks. -1 . For the area integrand $A(z)$ one has $h_{F}=0$, and (9) reduces to (3) for minimal surfaces. ${ }^{3}$ In the case of immersed surfaces with bounded mean curvature as critical points of the parametric functional with the integrand $E(y, z)$, the inequality (9) simplifies to the estimate (6), since $h_{F}=h$ in this situation.

2. For a geometric interpretation of the term $\left\|F_{y z}\right\|$ in the definition of $h_{F}$ in (8) we recall the notion of the $F$-mean curvature $H_{F}(X, N)=H_{F}:=-\operatorname{tr}\left(A_{F} S\right)$ and the $F$ Gau $\beta$ curvature $K_{F}(X, N)=K_{F}:=\operatorname{det}\left(A_{F} S\right)$, as introduced in [4]. Here, $S: T_{w} B \rightarrow$ $T_{w} B$ is the shape operator defined by $D X \circ S:=D N$ on the tangent space $T_{w} B$, and $A_{F}: T_{w} B \rightarrow T_{w} B$ is the symmetric endomorphism given by

$$
V \mapsto A_{F}(V):=(D X)^{-1}\left(F_{z z}(X, N) D X(V)\right) \text {. }
$$

For the special parametric integrands $A(z)=|z|$ and $E(y, z)=|z|+\langle Q(y), z\rangle$ the curvature functions $H_{F}$ and $K_{F}$ reduce to the classical mean curvature $H$ and Gauß

\footnotetext{
${ }^{2}$ In contrast to [7] and [8] we adopt the convention that the mean curvature $H$ is the sum of the principal curvatures, which accounts for the factor $1 / 2$ in the denominator in (6).

${ }^{3}$ The factor $4 \pi$ instead of 8 may be obtained using Wirtinger's Inequality without any smallness condition of the form (7), see e.g. [6, Ch. 6.3].
} 
curvature $K$, respectively, since one has $A_{A}=A_{E}=\operatorname{Id}_{T_{w} B}$. The first author proved in [3] that the Euler equation for $\mathcal{F}$ can be written as

$$
H_{F}=\sum_{i=1}^{3} F_{y^{i}} z^{i}(X, N)
$$

which shows that (9) may be regarded as an isoperimetric inequality for immersed $\mathcal{F}$ critical surfaces with bounded $F$-mean curvature. In the proof of Theorem 1 we actually work with $\left\|H_{F}\right\|_{\infty, B}$ instead of $\left\|F_{y z}\right\|$. Consequently, (9) is also valid for immersions that are not necessarily $\mathcal{F}$-critical but whose $F$-mean curvature $H_{F}$ is a prescribed bounded function on $\bar{B} \subset \mathbb{R}^{2}$, we merely have to replace the term $\left\|F_{y z}\right\|$ in (8) by $\left\|H_{F}\right\|_{\infty, B}$. Hence, the isoperimetric inequality in [7, Theorem $\left.1^{\prime}\right]$ is a special case of Theorem 1 above in the context of immersed surfaces.

Finally, for parametric integrands $F(y, z)=F(z)$ depending only on the $z$-variable, $\mathcal{F}$-critical immersions have vanishing $F$-mean curvature $H_{F}$. By a slight modification of the proof of Theorem 1 in Section 3 below, one obtains the isoperimetric inequality presented in [4].

3. The regularity assumption on $X$ (which also implies $\Gamma \in C^{2, \alpha}$ ) is due to the use of the Gauß-Bonnet Theorem. It is not clear whether one can relax the assumptions to $X \in C^{0}\left(\bar{B}, \mathbb{R}^{3}\right) \cap C^{2}\left(B, \mathbb{R}^{3}\right)$ and to closed curves $\Gamma$ that are merely rectifiable as in the case of surfaces with bounded mean curvature. In addition, it is an open question how to treat $\mathcal{F}$-critical surfaces with branch points for general parametric functionals.

We are going to prove an isoperimetric inequality slightly stronger than (9) using the radius $R_{\Gamma}(X)$ of the smallest ball in $\mathbb{R}^{3}$ containing the curve $\Gamma$, which can be expressed as

$$
R_{\Gamma}(X):=\inf _{q \in \mathbb{R}^{3}}\|X(.)-q\|_{\infty, \partial B} .
$$

THEOREM 2.- Under the assumptions of Theorem 1 the following isoperimetric inequality holds true:

$$
\mathcal{A}(X) \leqslant R_{\Gamma}(X) \cdot \frac{2 C(F) \frac{M_{2}}{M_{1}}\left[\int_{\Gamma} k d s-2 \pi\right]+\sqrt{\frac{M_{2}}{M_{1}}} L(\Gamma)}{2-R_{\Gamma}(X) h_{F}} .
$$

If the boundary curve $\Gamma$ is long in comparison to the radius $R_{\Gamma}(X)$, then the estimate (13) is better than (9), since it depends at most linearly on the length $L(\Gamma)$. Theorem 2 reduces to the linear isoperimetric inequality for minimal surfaces presented in [6, p. 388], because $h_{F}=C(F)=0$ and $M_{1}=M_{2}=1$ in that case. Notice that Theorem 1 follows from Theorem 2 by (7) and a simple geometric observation that leads to

$$
R_{\Gamma}(X) \leqslant \min \left\{1, \frac{L(\Gamma)}{4}\right\} .
$$

If $X$ minimizes the functional (1) within the class of surfaces bounded by $\Gamma$, one merely needs to assume that the continuous parametric integrand $F$ satisfies the growth 
condition

$$
m_{1}|z| \leqslant F(y, z) \leqslant m_{2}|z|
$$

for some constants $0<m_{1} \leqslant m_{2}<\infty$ without further regularity assumptions on $F$. Moreover, the minimizer $X$ does not have to be immersed, and it suffices to know that $X$ is in the Sobolev class $W^{1,2}\left(B, \mathbb{R}^{3}\right)$, and that $\Gamma$ is a rectifiable closed Jordan curve.

THEOREM 3.- Let $X \in W^{1,2}\left(B, \mathbb{R}^{3}\right)$ be a minimizer for the parametric functional (1) within the class of mappings $Z \in W^{1,2}\left(B, \mathbb{R}^{3}\right)$ such that the trace $\left.Z\right|_{\partial B}$ on $\partial B$ is $a$ continuous, weakly monotonic mapping ${ }^{4}$ of $\partial B$ onto a closed rectifiable Jordan curve $\Gamma$. Assume that the Lagrangian $F$ of class $C^{0}\left(\mathbb{R}^{3} \times \mathbb{R}^{3}\right)$ satisfies (15). Then

$$
\mathcal{A}(X) \leqslant \frac{m_{2}}{4 \pi m_{1}} L^{2}(\Gamma)
$$

Remark. - In this context no ellipticity condition is needed. In order to prove the existence of a minimizer for (1), however, one has to assume convexity of $F$ in the second argument, see [9,10].

To describe the strategy for proving Theorem 1 without getting involved with the technical details which arise in the general case, let us give a short proof of the isoperimetric inequality (6) for surfaces of bounded mean curvature. Such surfaces satisfy the partial differential equations

$$
\begin{gathered}
\Delta X=H(X) X_{u} \wedge X_{v}, \\
\left|X_{u}\right|^{2}=\left|X_{v}\right|^{2}, \quad\left\langle X_{u}, X_{v}\right\rangle=0
\end{gathered}
$$

on the domain $B$, where $H$ is a given bounded function on $\mathbb{R}^{3}$. Using (18) and integrating by parts we may write

$$
\begin{aligned}
\mathcal{A}(X) & =\mathcal{D}(X):=\frac{1}{2} \int_{B}|\nabla X(w)|^{2} d w \\
& \leqslant-\frac{1}{2} \int_{B}\langle\Delta X(w), X(w)-q\rangle+\frac{1}{2} \int_{\partial B}\left|X_{r}(w)\right| \cdot|X(1, \theta)-q| d \theta
\end{aligned}
$$

for any $q \in \mathbb{R}^{3}$, where for $w=(u, v)=r \mathrm{e}^{\mathrm{i} \theta} \in \bar{B}$ we have identified $X(w)$ with $X(r, \theta)$. We observe that there is a vector $q^{*} \in \mathbb{R}^{3}$ with $\left\|X(.)-q^{*}\right\|_{\infty, \partial B}=R_{\Gamma}(X) \leqslant 1$ by assumption (5). Inserting Eq. (17) into (19) we infer for $q=q^{*}$

$$
\begin{aligned}
\mathcal{A}(X) & \leqslant \frac{1}{2} \int_{B}\left|H(X(w))\left\|X(w)-q^{*}|| X_{u} \wedge X_{v} \mid d w+\frac{1}{2}\right\| X(.)-q^{*} \|_{\infty, \partial B} L(\Gamma)\right. \\
& \leqslant \frac{1}{2} h R_{\Gamma}(X) \mathcal{A}(X)+\frac{1}{2} R_{\Gamma}(X) L(\Gamma),
\end{aligned}
$$

\footnotetext{
${ }^{4}$ See $[6$, Ch. 4.2] for the notion of weakly monotonic mappings on the boundary.
} 
where we have used that $\left|X_{\theta}\right|=\left|X_{r}\right|$ on $\partial B$ by (18), and the fact that

$$
\left\|X(.)-q^{*}\right\|_{\infty, B} \leqslant\left\|X(.)-q^{*}\right\|_{\infty, \partial B}
$$

by an application of the maximum principle for (17). In fact, one can show that the function $f(w):=\left|X(w)-q^{*}\right|^{2}$ is a subsolution for the Laplace operator under the smallness assumption (5), see [7, Lemma 1]. With (14) we arrive at (6).

In Section 2 we introduce the tools necessary to take up the approach outlined above. Proposition 2.2 gives the inclusion principle generalizing (20), the conformality relations are suitably adapted to the general parametric integrand $F$ in (29), (30). The partial differential equation (17) will be replaced by a differential inequality in Proposition 2.4 containing first derivatives of $X$ and of its normal $N$ on the right-hand side. Therefore it is necessary to estimate the Dirichlet energy $\mathcal{D}(N)$ of the normal of an $\mathcal{F}$-critical immersion, which will be done using the Gauß-Bonnet Theorem, see Lemma 2.5. Section 3 contains the proof for Theorem 2 along the lines of the arguments described above, as well as a short proof of Theorem 3.

\section{Inclusion theorem and $\boldsymbol{F}$-conformal parameters}

Let $X: M \hookrightarrow \mathbb{R}^{3}$ be an immersion of an orientable smooth manifold $M$ of dimension 2 into $\mathbb{R}^{3}$, where $X \in C^{2}\left(M, \mathbb{R}^{3}\right)$. We are going to work with the induced metric

$$
g(V, W):=\langle D X(V), D X(W)\rangle \text { for } V, W \in T_{p} M
$$

and the globally well-defined normal mapping $N: M \rightarrow S^{2}$. Consider the parametric variational integral

$$
\mathcal{F}(X):=\int_{M} F(X, N) d A
$$

with an elliptic parametric Lagrangian $F=F(y, z) \in C^{0}\left(\mathbb{R}^{3} \times \mathbb{R}^{3}\right) \cap C^{3, \alpha}\left(\mathbb{R}^{3} \times\left(\mathbb{R}^{3}-\right.\right.$ $\{0\})$ ) satisfying the homogeneity condition $(\mathrm{H})$.

As a starting point we give a generalization of the well-known identity $\Delta_{M} X=H N$, where $\Delta_{M}=\operatorname{div}_{M} \operatorname{grad}_{M}$ is the Laplace-Beltrami operator associated to $M$. To this end we introduce the differential operator

$$
\theta_{F}[\varphi]:=\operatorname{div}_{M}\left(A_{F} \operatorname{grad}_{M} \varphi\right)-\left(\operatorname{div}_{M} A_{F}\right)[\varphi] \text { for } \varphi \in C^{2}(M),
$$

where $A_{F}$ is defined in (10) of the introduction. In [3] the following result is proved:

THEOREM 2.1. - Let $X \in C^{2}\left(M, \mathbb{R}^{3}\right)$ be an immersion with normal $N$ and $F$-mean curvature $H_{F}$. Then we have

$$
\theta_{F} X=H_{F} N
$$

Let us point out that according to the ellipticity condition (E) the eigenvalues of $F_{z z}(y, z): z^{\perp} \rightarrow z^{\perp}$ are bounded by $M_{1}$ and $M_{2}$ for $|y| \leqslant 1$ in the case of elliptic integrands as definied in (E). Obviously, $\theta_{F}$ is an elliptic operator, if $F$ is elliptic. 
This allows us to prove the following inclusion principle for $\mathcal{F}$-critical immersions of a smooth manifold $M$ with boundary $\partial M$, where we set $\bar{M}:=M \cup \partial M$.

PROPOSITION 2.2. - Let $F$ be an elliptic integrand and $X$ be an immersion of class $C^{2}\left(M, \mathbb{R}^{3}\right) \cap C^{0}\left(\bar{M}, \mathbb{R}^{3}\right)$ such that

$$
X(\bar{M}) \subset \overline{B_{1}(0)} \subset \mathbb{R}^{3}, \text { and }\left\|H_{F}\right\|_{\infty, M}<2 M_{1} .
$$

Then $X(\partial M) \subset \overline{B_{r}(p)}$ for some $p \in \mathbb{R}^{3}$ and some radius $0<r \leqslant 1$, implies $X(\bar{M}) \subset$ $\overline{B_{r}(p)}$.

Proof. - We apply a continuity method as in [7]. For $\sigma \in[0,1]$ we consider the family of surfaces $X_{\sigma}(w):=X(w)-\sigma p$ and note that by our assumptions (23) one has

$$
\left\|X_{\sigma}(.)\right\|_{\infty, \partial M}=\|\sigma(X(.)-p)+(1-\sigma) X(.)\|_{\infty, \partial M} \leqslant 1 .
$$

Using the chain rule we calculate on $M$

$$
\begin{aligned}
\theta_{F} X_{\sigma}^{2}= & 2 \sum_{i=1}^{3} X_{\sigma}^{i} \operatorname{div}_{M}\left(A_{F} \operatorname{grad}_{M}\left(X^{i}\right)\right) \\
& +2 \sum_{i=1}^{3} g\left(\operatorname{grad}_{M}\left(X_{\sigma}^{i}\right), A_{F} \operatorname{grad}_{M}\left(X^{i}\right)\right)-2 \sum_{i=1}^{3} X_{\sigma}^{i}\left(\operatorname{div}_{M} A_{F}\right)\left(X^{i}\right) \\
= & 2 \sum_{i=1}^{3} X_{\sigma}^{i} \theta_{F} X^{i}+2 \sum_{i=1}^{3} g\left(\operatorname{grad}_{M}\left(X_{\sigma}^{i}\right), A_{F} \operatorname{grad}_{M}\left(X^{i}\right)\right) \\
\underset{(22)}{=} & 2 H_{F} \sum_{i=1}^{3} X_{\sigma}^{i} N^{i}+2 \sum_{i=1}^{3} g\left(\operatorname{grad}_{M}\left(X^{i}\right), A_{F} \operatorname{grad}_{M}\left(X^{i}\right)\right) \\
\geqslant & -2\left\|H_{F}\right\|_{\infty, M}\left\|X_{\sigma}(.)\right\|_{\infty, M}+4 M_{1} .
\end{aligned}
$$

If the condition

$$
\left\|H_{F}\right\|_{\infty, M}\left\|X_{\sigma}(.)\right\|_{\infty, M} \leqslant 2 M_{1}
$$

holds true for every $\sigma \in[0,1]$, then $X_{\sigma}^{2}$ and in particular $X_{1}^{2}(w)=(X(w)-p)^{2}$ is a subsolution for the elliptic operator $\theta_{F}$, which implies by the maximum principle the statement of the proposition. The condition (25) is certainly valid for each $\sigma \in[0,1]$ if $\left\|H_{F}\right\|_{\infty, M}=0$. Hence by virtue of (23) we may assume that $0<\left\|H_{F}\right\|_{\infty, M}<2 M_{1}$. Then one has either

$$
\left\|X_{\sigma}(.)\right\|_{\infty, M}>\frac{2 M_{1}}{\left\|H_{F}\right\|_{\infty, M}}=: c_{1} \underset{(23)}{>} 1,
$$

or (25) is true, which implies according to (24) and the maximum principle

$$
\left\|X_{\sigma}(.)\right\|_{\infty, M} \leqslant 1 \text {. }
$$

Note that the function $\sigma \mapsto\left\|X_{\sigma}(.)\right\|_{\infty, M}$ is continuous on [0,1], and that $\left\|X_{0}(.)\right\|_{\infty, M} \leqslant$ 1. If there were some $\sigma_{1} \in(0,1]$ with (26), i.e., with $\left\|X_{\sigma_{1}}(.)\right\|_{\infty, M}>c_{1}>1$, then 
by the intermediate value theorem there would be some parameter $\sigma_{2} \in\left(0, \sigma_{1}\right)$ with $\left\|X_{\sigma_{2}}(.)\right\|_{\infty, M}=c_{1}$. But this would contradict our observation that for any $\sigma \in[0,1]$ either (26) or (27) must hold. Consequently, (26) is not possible for any $\sigma \in[0,1]$, which proves (25).

From now on we focus on immersions $X: B \rightarrow \mathbb{R}^{3}$ of $B \subset \mathbb{R}^{2}$ into $\mathbb{R}^{3}$ of class $C^{2}\left(B, \mathbb{R}^{3}\right)$, where $B$ is the open unit disk in $\mathbb{R}^{2}$. In this case a special parametrization simplifies the situation. We introduce so-called $F$-conformal parameters defined via the metric

$$
g_{F}(V, W):=g\left(A_{F}^{-1} V, W\right) \text { for } V, W \in T_{x} B
$$

as follows. An immersion $X(u, v): B \rightarrow \mathbb{R}^{3}$ is given in $F$-conformal parameters $w=$ $(u, v)$, if $g_{F}$ is diagonalized, i.e.,

$$
\begin{gathered}
g_{F}(w)\left(\frac{\partial}{\partial u}, \frac{\partial}{\partial u}\right)=g_{F}(w)\left(\frac{\partial}{\partial v}, \frac{\partial}{\partial v}\right)=W_{F}, \\
g_{F}(w)\left(\frac{\partial}{\partial u}, \frac{\partial}{\partial v}\right)=0 \quad \text { for all } w \in B .
\end{gathered}
$$

The following simple result from planar linear algebra proven in [4] turns out to be useful for computing the conformal factor $W_{F}$ and for other calculations in $F$-conformal parameters:

LEMMA 2.3. - Let $V$ be a two-dimensional vector space with an inner product. If $\psi: V \rightarrow V$ is a positive definite symmetric endomorphism and $D^{90}: V \rightarrow V$ a rotation about an angle of $90^{\circ}$, then $D^{90} \circ \psi=(\operatorname{det} \psi) \psi^{-1} \circ D^{90}$.

Remark. - The lemma will be applied to the rotation $D(w): N(w)^{\perp} \rightarrow N(w)^{\perp}$ defined by $D(w) Z:=N(w) \wedge Z$ for $Z \in N(w)^{\perp}$.

Using the notation $\Phi(w):=F_{z z}(X(w), N(w)): N(w)^{\perp} \rightarrow N(w)^{\perp}$ the $F$-conformality relations may be rewritten as

$$
\begin{aligned}
0<W_{F} & =\left\langle X_{u}, \Phi^{-1} X_{u}\right\rangle=\left\langle X_{v}, \Phi^{-1} X_{v}\right\rangle, \\
0 & =\left\langle X_{v}, \Phi^{-1} X_{u}\right\rangle .
\end{aligned}
$$

Thus we can write with Lemma 2.3

$$
\begin{aligned}
& X_{u}=\mu_{2}\left(\left(\Phi^{-1} X_{v}\right) \wedge N\right)=\mu_{2} \Phi(\operatorname{det} \Phi)^{-1}\left(X_{v} \wedge N\right), \\
& X_{v}=\mu_{1}\left(N \wedge\left(\Phi^{-1} X_{u}\right)\right)=\mu_{1} \Phi(\operatorname{det} \Phi)^{-1}\left(N \wedge X_{u}\right)
\end{aligned}
$$

for some numbers $\mu_{1}, \mu_{2} \in \mathbb{R}$. Therefore one obtains for the conformal factor $W_{F}$

$$
0<W_{F}=\left\langle\Phi^{-1} X_{v}, X_{v}\right\rangle=\mu_{2}(\operatorname{det} \Phi)^{-1}\left\langle N \wedge X_{u}, X_{v}\right\rangle=\mu_{2}(\operatorname{det} \Phi)^{-1} W,
$$

where $W:=\left|X_{u} \wedge X_{v}\right|$. Furthermore we have $W N=X_{u} \wedge X_{v}=\mu_{1} W_{F} N=\mu_{2} W_{F} N$. Consequently, we get $\mu_{1}=\mu_{2}=W / W_{F}$, and then

$$
W_{F}=W / \sqrt{\operatorname{det} \Phi}, \quad \mu_{1}=\mu_{2}=\sqrt{\operatorname{det} \Phi} .
$$


The following calculation will lead to an analytic interpretation of the $F$-mean curvature of an $F$-conformally parametrized surface $X: B \rightarrow \mathbb{R}^{3}$. By the definition of the $F$-mean curvature we have

$$
H_{F}=-\operatorname{tr}\left(A_{F} S\right)=-g^{\alpha \beta} g\left(\frac{\partial}{\partial u^{\alpha}}, A_{F} S \frac{\partial}{\partial u^{\beta}}\right)=-g^{\alpha \beta}\left\langle X_{u^{\alpha}}, F_{z z}(X, N) N_{u^{\beta}}\right\rangle .
$$

The two identities $N \wedge X_{u}=W g^{2 \alpha} X_{u^{\alpha}}, N \wedge X_{v}=-W g^{1 \alpha} X_{u^{\alpha}}$ are used to obtain

$$
\begin{aligned}
H_{F} & =\left(\left\langle N \wedge X_{v}, F_{z z}(X, N) N_{u}\right\rangle-\left\langle N \wedge X_{u}, F_{z z}(X, N) N_{v}\right\rangle\right) / W \\
& =\left(\left\langle\Phi\left(N \wedge X_{v}\right), N_{u}\right\rangle-\left\langle\Phi\left(N \wedge X_{u}\right), N_{v}\right\rangle\right) / W \\
& =-\sqrt{\operatorname{det} \Phi}\left(\left\langle X_{u}, N_{u}\right\rangle+\left\langle X_{v}, N_{v}\right\rangle\right) / W \\
\underset{(32)(31)}{=} & \langle\Delta X, N\rangle / W_{F} .
\end{aligned}
$$

Thus we have found that the normal component of $\Delta X$ is given by $H_{F} W_{F} N$, if $X$ is an immersion in $F$-conformal parameters.

For technical reasons we introduce the linear mapping

$$
l(y, z):=\frac{F_{z z}(y, z)}{\sqrt{\left.\operatorname{det} F_{z z}(y, z)\right|_{z^{\perp}}}}+\frac{z}{|z|} \otimes \frac{z}{|z|},
$$

where $y \in \mathbb{R}^{3}, z \in \mathbb{R}^{3}-\{0\}$. Note that we can rewrite (31), (32) as

$$
\begin{aligned}
& X_{u}=l(X, N)\left(X_{v} \wedge N\right), \\
& X_{v}=l(X, N)\left(N \wedge X_{u}\right),
\end{aligned}
$$

since the wedge products on the right-hand side are tangential vectors in $N^{\perp}$. Differentiating these equations we arrive at

$$
\begin{aligned}
\Delta X= & (l(X, N))_{u}\left(X_{v} \wedge N\right)+(l(X, N))_{v}\left(N \wedge X_{u}\right) \\
& +l(X, N)\left(X_{v} \wedge N_{u}+N_{v} \wedge X_{u}\right) .
\end{aligned}
$$

The tangential part of $\Delta X$ may be estimated by

$$
\left|(\Delta X)^{\tan }\right| \leqslant\left|(l(X, N))_{u}\left(X_{v} \wedge N\right)+(l(X, N))_{v}\left(N \wedge X_{u}\right)\right|,
$$

whereas the normal part, according to (34), is given by

$$
\left|(\Delta X)^{\mathrm{nor}}\right|=|\langle\Delta X, N\rangle|=\left|H_{F}\right| W_{F} .
$$

A simple application of the chain rule leads to an a priori estimate of $\Delta X$ :

$$
|\Delta X| \leqslant 2\left\|l_{y}\right\|\left|X_{u}\right|\left|X_{v}\right|+\left\|l_{z}\right\|\left(\left|X_{u}\right|\left|N_{v}\right|+\left|X_{v}\right|\left|N_{u}\right|\right)+\left|H_{F}\right| W_{F} .
$$

Remark. - Immersed surfaces of bounded mean curvature $H$ may be considered as critical points of the parametric functional with the integrand $E(y, z)$ defined in (4) in 
the introduction. Note that the $F$-conformality relations (29), (30) reduce to the classical conformality relations (18) mentioned in the introduction, since $A_{F}=\operatorname{Id}_{T_{w} B}$ in that case. Moreover, $(l(X, N))_{u}=(l(X, N))_{v}=0$ and (36) together with (34) reduce to the wellknown differential equation (17) given in the introduction.

Let us summarize (34) and (38) in

Proposition 2.4. - Let $X: B \rightarrow \mathbb{R}^{3}$ be an $F$-conformally parametrized immersion of class $C^{2}\left(B, \mathbb{R}^{3}\right)$. Then

(i) $\langle\Delta X, N\rangle=H_{F} W_{F}$.

(ii) $|\Delta X| \leqslant C_{y}(F)\left|X_{u}\right|\left|X_{v}\right|+C_{z}(F)\left(\left|X_{u}\right|\left|N_{v}\right|+\left|X_{v}\right|\left|N_{u}\right|\right)+\left|H_{F}\right| W_{F}$, where $C_{y}, C_{z}$ are constants depending only on the integrand $F$.

Note that $C_{y}, C_{z}$ can be estimated from above in terms of $\|F\|_{C^{3}\left(\overline{B_{1}(0)} \times S^{2}\right)}$ and $M_{1}$, if $X(\bar{B}) \subset \overline{B_{1}(0)}$.

The theorem of Cayley-Hamilton applied to the endomorphism $A_{F} S: T_{w} B \rightarrow T_{w} B$ leads to the algebraic relation

$$
S A_{F} S+K_{F} A_{F}^{-1}+H_{F} S=0 .
$$

This gives us a gradient estimate for the normal $N$ :

$$
\begin{aligned}
|\nabla N|^{2} \leqslant & \frac{1}{M_{1}}\left[\left\langle\Phi N_{u}, N_{u}\right\rangle+\left\langle\Phi N_{v}, N_{v}\right\rangle\right] \\
= & \frac{1}{M_{1}}\left[g\left(S A_{F} S\left(\frac{\partial}{\partial u}\right), \frac{\partial}{\partial u}\right)+g\left(S A_{F} S\left(\frac{\partial}{\partial v}\right), \frac{\partial}{\partial v}\right)\right] \\
= & \frac{-1}{M_{1}}\left[H_{F}\left\{\left\langle D X \circ S\left(\frac{\partial}{\partial u}\right), D X\left(\frac{\partial}{\partial u}\right)\right\rangle+\left\langle D X \circ S\left(\frac{\partial}{\partial v}\right), D X\left(\frac{\partial}{\partial v}\right)\right\rangle\right\}\right. \\
& \left.+K_{F}\left\{g\left(A_{F}^{-1}\left(\frac{\partial}{\partial u}\right), \frac{\partial}{\partial u}\right)+g\left(A_{F}^{-1}\left(\frac{\partial}{\partial v}\right), \frac{\partial}{\partial v}\right)\right\}\right] \\
= & -\frac{1}{M_{1}}\left[H_{F}\left\{\left\langle N_{u}, X_{u}\right\rangle+\left\langle N_{v}, X_{v}\right\rangle\right\}+K_{F}\left\{g_{F}\left(\frac{\partial}{\partial u}, \frac{\partial}{\partial u}\right)+g_{F}\left(\frac{\partial}{\partial v}, \frac{\partial}{\partial v}\right)\right\}\right] \\
= & \frac{W_{F}}{M_{1}}\left[H_{F}^{2}-2 K_{F}\right],
\end{aligned}
$$

where we have used (i) of Proposition 2.4. Now we are in the position to deduce a geometric estimate for the Dirichlet energy

$$
\mathcal{D}(N):=\frac{1}{2} \int_{B}|\nabla N(w)|^{2} d w
$$

of the normal $N$ of an $F$-conformal immersion $X$.

LEMMA 2.5. - The normal $N$ of an $F$-conformal immersion $X \in C^{2}\left(\bar{B}, \mathbb{R}^{3}\right)$ with $X(\bar{B}) \subset \overline{B_{1}(0)}$ satisfies

$$
\mathcal{D}(N) \leqslant \frac{M_{2}}{M_{1}}\left[\int_{\Gamma} \kappa_{g} d s-2 \pi\right]+\frac{M_{2}}{2 M_{1}^{3}} \int_{X} H_{F}^{2} d A .
$$


Proof. - We may write

$$
K_{F}=\operatorname{det}\left(A_{F} S\right)=\operatorname{det} \Phi \operatorname{det} S=K \operatorname{det} \Phi .
$$

From (33), (40), and the ellipticity condition (E) we infer

$$
\begin{aligned}
\frac{M_{1}}{M_{2}}|\nabla N|^{2} & \underset{(40)}{\leqslant} \frac{W_{F}}{(\operatorname{det} \Phi)^{1 / 2}}\left[H_{F}^{2}-2 K_{F}\right] \\
& =\frac{W}{(33),(42)} H_{F}^{2}-2 K W \\
& \leqslant \frac{W}{M_{1}^{2}} H_{F}^{2}-2 K W .
\end{aligned}
$$

The estimate (41) follows now by integrating this inequality over the domain $B$ and applying the Gauß-Bonnet Theorem.

\section{Proofs of the main results}

Proof of Theorem 2. - Since (9) is a purely geometric estimate, we may assume that $X$ is given in $F$-conformal parameters. If this is not the case one may apply the uniformization theorem in a version proved by Sauvigny [11, Thm. 2] to the $C^{1, \alpha}$-metric (28), or apply our argument in $[4$, p. 94] to find a positively oriented diffeomorphism $w: \bar{B} \rightarrow \bar{B}$ of class $C^{2, \alpha}\left(\bar{B}, \mathbb{R}^{2}\right)$, such that $X \circ w^{-1} \in C^{2, \alpha}\left(\bar{B}, \mathbb{R}^{3}\right)$ is $F$-conformal. Integrating by parts we may estimate the Dirichlet energy of an $F$-conformal immersion for any $q \in \mathbb{R}^{3}$ as

$$
\begin{aligned}
\mathcal{D}(X) & =\frac{1}{2} \int_{B}\langle D X(w), D(X(w)-q)\rangle d w \\
& \leqslant \frac{1}{2} \int_{B}|\Delta X(w)||X(w)-q| d w+\frac{1}{2} \int_{\partial B}\left|X_{r}(1, \theta) \| X(1, \theta)-q\right| d \theta,
\end{aligned}
$$

where for $w=(u, v)=r \mathrm{e}^{\mathrm{i} \theta} \in \bar{B}$ we have identified $X(w)$ and $X(r, \theta)$. Using Proposition 2.4(ii) and Lemma 2.5 we obtain

$$
\begin{aligned}
\mathcal{D}(X) \leqslant & \frac{1}{4} C_{y}(F) \int_{B}|\nabla X(w)|^{2}|X(w)-q| d w \\
& +\frac{1}{2} C_{z}(F) \int_{B}\left[\left|X_{u}(w)\right|\left|N_{v}(w)\right|+\left|X_{v}(w)\right|\left|N_{u}(w)\right|\right] \cdot|X(w)-q| d w \\
& +\frac{1}{2} \int_{B}\left|H_{F}(X(w), N(w))\right||X(w)-q| W_{F}(w) d w \\
& +\frac{1}{2} \int_{\partial B}\left|X_{r}(1, \theta)\right||X(1, \theta)-q| d \theta
\end{aligned}
$$




$$
\begin{aligned}
\underset{(33)}{\leqslant} & {\left[\frac{C_{y}(F)}{2}+\frac{C_{z}(F)}{2}+\frac{\left\|H_{F}\right\|_{\infty, B}}{2 M_{1}}\right]\|X-q\|_{\infty, B} \mathcal{D}(X) } \\
& +\frac{C_{z}(F)}{2}\|X-q\|_{\infty, B} \mathcal{D}(N)+\frac{1}{2} \int_{\partial B}\left|X_{r}(1, \theta) \| X(1, \theta)-q\right| d \theta \\
\underset{(41)}{\leqslant} & {\left[\frac{C_{y}(F)}{2}+\frac{C_{z}(F)}{2}+\frac{\left\|H_{F}\right\|_{\infty, B}}{2 M_{1}}+C_{z}(F) \frac{M_{2}}{4 M_{1}^{3}}\left\|H_{F}\right\|_{\infty, B}^{2}\right]\|X-q\|_{\infty, B} \mathcal{D}(X) } \\
& +\frac{C_{z}(F)}{2}\|X-q\|_{\infty, B} \frac{M_{2}}{M_{1}}\left[\int_{\Gamma} \kappa_{g} d s-2 \pi\right] \\
& +\frac{1}{2} \int_{\partial B}\left|X_{r}(1, \theta) \| X(1, \theta)-q\right| d \theta .
\end{aligned}
$$

Defining

$$
C(F):=C_{y}(F)+C_{z}(F)\left(1+\frac{M_{2}}{2 M_{1}^{3}}\right),
$$

we insert the Euler equation (11) to arrive at

$$
\begin{aligned}
\mathcal{D}(X) \leqslant & \frac{1}{2}\left[C(F)\left(1+\left\|F_{y z}\right\|^{2}\right)+\frac{\left\|F_{y z}\right\|}{M_{1}}\right]\|X-q\|_{\infty, B} \mathcal{D}(X) \\
& +\|X-q\|_{\infty, B} C(F) \frac{M_{2}}{M_{1}}\left[\int_{\Gamma} \kappa_{g} d s-2 \pi\right] \\
& +\frac{1}{2}\|X-q\|_{\infty, \partial B} \sqrt{\frac{M_{2}}{M_{1}}} L(\Gamma),
\end{aligned}
$$

for any $q \in \mathbb{R}^{3}$, where we have used the ellipticity condition (E) and the conformality relations (29) and (30) in polar coordinates for the boundary integral, i.e. for $|w|=r=1$ :

$$
\left|X_{r}\right|^{2} \leqslant M_{2}\left\langle X_{r}, \Phi^{-1} X_{r}\right\rangle=M_{2}\left\langle X_{\theta}, \Phi^{-1} X_{\theta}\right\rangle \leqslant \frac{M_{2}}{M_{1}}\left|X_{\theta}\right|^{2} .
$$

Note that $C(F)$ can be estimated from above in terms of $\|F\|_{C^{3}\left(\overline{B_{1}(0)} \times S^{2}\right)}$ and $M_{1}$. We notice that by assumption (7) there is a vector $q^{*} \in \mathbb{R}^{3}$, such that $\left\|X(.)-q^{*}\right\|_{\infty, \partial B}=$ $R_{\Gamma}(X) \leqslant 1$, where $R_{\Gamma}(X)$ is defined in (12) in the introduction. By (7) and (8) we may apply the inclusion principle Proposition 2.2 to conclude $\left\|X(.)-q^{*}\right\|_{\infty, \bar{B}} \leqslant R_{\Gamma}(X)$. These observations together with (43) for $q=q^{*}$ lead to the desired result, since then we may write

$$
\mathcal{D}(X) \leqslant R_{\Gamma}(X) \cdot \frac{2 C(F) \frac{M_{2}}{M_{1}}\left[\int_{\Gamma} \kappa_{g} d s-2 \pi\right]+\sqrt{\frac{M_{2}}{M_{1}}} L(\Gamma)}{2-R_{\Gamma}(X)\left[C(F)\left(1+\left\|F_{y z}\right\|^{2}\right)+\frac{\left\|F_{y z}\right\|}{M_{1}}\right]} .
$$

Proof of Theorem 3. - Let $Y$ be a disk-type minimal surface bounded by the curve $\Gamma$. Then the classical isoperimetric inequality (3) and the growth condition (15) imply that 
for the minimizer $X$ we can conclude $m_{1} \mathcal{A}(X) \leqslant \mathcal{F}(X) \leqslant \mathcal{F}(Y) \leqslant m_{2} \mathcal{A}(Y) \leqslant \frac{m_{2}}{4 \pi} L^{2}(\Gamma)$, which proves the result.

Remark. - Using an isoperimetric inequality for harmonic vector functions due to Morse and Tompkins, see e.g. [5, pp. 135-138], one may use a similar comparison argument to prove a local version of (16) for minimizers:

$$
\mathcal{A}_{\Omega}(X):=\int_{\Omega}\left|X_{u} \wedge X_{v}\right| d u d v \leqslant \frac{m_{2}}{4 m_{1}} L^{2}(X(\partial \Omega))
$$

for all simply connected subdomains $\Omega \subset B$ whose boundary is piecewise smooth. In fact, one uses the comparison surface

$$
Z:= \begin{cases}Y & \text { on } \Omega \\ X & \text { on } \bar{B}-\Omega\end{cases}
$$

where $Y \in C^{0}\left(\bar{\Omega}, \mathbb{R}^{3}\right) \cap C^{2}\left(\Omega, \mathbb{R}^{3}\right)$ satisfies $\Delta Y=0$ on $\Omega$, and $X-Y \in W_{0}^{1,2}\left(\Omega, \mathbb{R}^{3}\right)$.

\section{REFERENCES}

[1] J.L. Barbosa, M. do Carmo, A proof of a general isoperimetric inequality for surfaces, Math. Z. 162 (3) (1978) 245-261.

[2] E.F. Beckenbach, T. Radó, Subharmonic functions and surfaces of negative curvature, Trans. Am. Math. Soc. 35 (1933) 662-664.

[3] U. Clarenz, Enclosure theorems for extremals of elliptic parametric functionals, Calc. Var., online publication DOI 10.1007/s005260100128, 2001.

[4] U. Clarenz, H. von der Mosel, Compactness theorems and an isoperimetric inequality for critical points of elliptic parametric functionals, Calc. Var. 12 (2001) 85-107.

[5] R. Courant, Dirichlet's Principle, Conformal Mapping, and Minimal Surfaces, Interscience, New York, 1950.

[6] U. Dierkes, S. Hildebrandt, A. Küster, O. Wohlrab, Minimal Surfaces, Vol. I, Grundlehren math. Wiss., Vol. 295, Springer, Berlin, 1992.

[7] E. Heinz, S. Hildebrandt, The number of branch points of surfaces of bounded mean curvature, J. Differential Geom. 4 (1970) 227-235.

[8] S. Hildebrandt, Randwertprobleme für Flächen mit vorgeschriebener mittlerer Krümmung und Anwendungen auf die Kapillaritätstheorie, Math. Z. 112 (1969) 205-213.

[9] S. Hildebrandt, H. von der Mosel, On two-dimensional variational problems, Calc. Var. 9 (1999) 249-267.

[10] S. Hildebrandt, H. von der Mosel, Plateau's problem for parametric double integrals: I. Existence and regularity in the interior, Preprint 88 MPI f. Math. Leipzig, 2001. Preprint 745 SFB 256 Univ. Bonn, 2001, to appear in Comm. Pure. Appl. Math.

[11] F. Sauvigny, Introduction of isothermal parameters into a Riemannian metric by the continuity method, Analysis 19 (1999) 235-243. 